\title{
Smart and advanced biomaterials for regenerative medicine and tissue engineering
}

\section{Biomateriales inteligentes y avanzados para la medicina regenerativa y la ingeniería de tejidos}

\section{Alvaro $\mathrm{Mata}^{1}$}

1 School of Engineering \& Materials Science, Queen Mary, University of London, Mile End Road, London E1 4NS, UK. Email: a.mata@qmul.ac.uk

\begin{abstract}
There is great interest to develop new materials with properties that resemble those of biological systems such as hierarchical organization, the capacity to grow or self-heal, and the ability to guide complex biological processes. These kinds of materials would open opportunities to engineer tissues with a much-needed higher level of complexity and overcome major obstacles in regenerative medicine. To this end, supramolecular chemistry offers an exciting opportunity to grow such materials from the bottomup using molecules and processes found in nature. However, the ability to transform molecular and nano-scale design into functional devices with practical utility at the macroscale remains a challenge.

The paper will describe new strategies that integrate supramolecular chemistry with engineering principles to develop practical materials with tuneable and advanced properties such as hierarchical organization, the capacity to grow, tuneable mechanical properties, and specific bioactivity (InostrozaBrito et al. 2015; Aguilar et al. 2017; Elsharkawy et al. 2018; Hedegaard et al. 2018). These materials are being used towards new regenerative therapies of tissues such as enamel, bone, and blood vessels as well as creating more biologically relevant in vitro models.
\end{abstract}

\section{References}

Aguilar, J.P., Lipka, M., Primo, G.A., Licon-Bernal, E.E., Fernández-Pradas, J.M., Yaroshchuk, A., Albericio, F. \& Mata, A. 2017. 3D Electrophoresis-Assisted Lithography (3DEAL): 3D molecular printing to create functional patterns and anisotropic hydrogels. Adv. Funct. Mater. 28(15): 1703014. DOI: 10.1002/adfm.201703014

Elsharkawy, S., Al-Jawad, M., Pantano, M.F., Tejeda-Montes, E., Mehta, K., Jamal, H., Agarwal, S., Shuturmiska, K., Rice, A., Tarakina, N.V., Wilson, R.M., Bushby, A.J., Alonso, M., RodríguezCabello, J.C., Barbieri, E., Hernández, A.d.R., Stevens, M.M., Pugno, N.M., Anderson, P. \& Mata, A. 2018. Protein disorder-order interplay to guide the growth of hierarchical mineralized structures. Nat. Commun. 9(2145): 1-12. DOI: 10.1038/s41467-018-04319-0 
Ciencias

Veterinarias

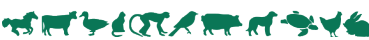

Rev. Ciencias Veterinarias, Vol. 37, Nº 3, [25-26], E-ISSN: 2215-4507

First Symposium on Regenerative Medicine and Nanotechnology, Costa Rica 2019 DOI: https://doi.org/10.15359/rcv.37-3.8

URL: http://www.revistas.una.ac.cr/index.php/veterinaria/index

Hedegaard, C.L., Collin, E.C., Redondo-Gómez, C., Nguyen, L.T.H., Ng, K.W., Castrejón-Pita, A.A., Castrejón-Pita, J.R. \& Mata, A. 2018. Hydrodynamically guided hierarchical self-assembly of peptide-protein bioinks. Adv. Funct. Mater. 28(16): 1703716. DOI: 10.1002/adfm.201703716

Inostroza-Brito, K.E., Collin, E., Siton-Mendelson, O., Smith, K.H., Monge-Marcet, A., Ferreira, D.S., Rodríguez, R.P., Alonso, M., Rodríguez-Cabello, J.C., Reis, R.L., Sagués, F., Botto, L., Bitton, R., Azevedo, H.S. \& Mata, A. 2015. Co-assembly, spatiotemporal control and morphogenesis of a hybrid protein-peptide system. Nat. Chem. 7(11): 897-904. DOI: 10.1038/nchem.2349 